\title{
Hopf bifurcations in resonance 2:1
}

\author{
A.M. Krasnosel'skii ${ }^{\mathrm{a}, 1,2}$ D.I.Rachinskii ${ }^{\mathrm{a}, 1,2,3}$ K. Schneider ${ }^{\mathrm{b}, 1}$ \\ ${ }^{a}$ Institute for Information Transmission Problems, \\ 19 Bolshoi Karetny Lane, Moscow 101447, Russia \\ ${ }^{\mathrm{b}}$ Weierstrass Institute for Applied Analysis and Stochastics, \\ Mohrenstrasse 39, D-10117 Berlin, Germany
}

Key words: Hopf bifurcation, Strong resonance, Degree theory, Control theory 1991 MSC: 34C23, 34C $35,54 \mathrm{H} 25$

\section{Introduction}

In this paper Hopf bifurcations for equations of control theory are studied in strong resonance case 2:1. The harmonic linearization approach and topological methods allow to give the sharp analysis of the problem for the case of quadratic principal nonlinearity.

Consider the equation

$$
L\left(\frac{d}{d t} ; \lambda\right) x=M\left(\frac{d}{d t} ; \lambda\right) f(x, \lambda)
$$

with the scalar parameter $\lambda$. Here

$$
L(p ; \lambda)=p^{\ell}+a_{1}(\lambda) p^{\ell-1}+\ldots+a_{\ell}(\lambda), \quad M(p ; \lambda)=b_{0}(\lambda) p^{m}+b_{1}(\lambda) p^{m-1}+\ldots+b_{m}(\lambda)
$$

are polynomials of degrees $\ell$ and $m$, let $\ell>m$. Equation (1) is usual in control theory (see, e.g., [1-3]), it describes the dynamics of a single-loop control system with a linear link with a rational transfer function $M(p ; \lambda) / L(p ; \lambda)$

1 E-mails: amk@iitp.ru, rach@iitp.ru, schneide@wias-berlin.de

2 A.M. Krasnosel'skii and D.I. Rachinskii were partially supported by Grants $N$ oㅡㅇㅡ 0001-00571 and 00-15-96116 of the Russian Foundation for Basic Researches. The research was done while A.M. Krasnosel'skii and D.I. Rachinskii were visiting the Weierstrass Institute.

3 D.I. Rachinskii was supported by the Research Fellowship from the Alexander von Humboldt Foundation. 
and nonlinear feedback $f(x, \lambda)$. If $M \equiv 1$, then (1) is an ordinary autonomous higher-order differential equation. The standard definition of solutions (for fixed $\lambda$ ) of equation (1) looks as follows. For any $L, M$, and $f(x)=f(x, \lambda)$ there exists a representation $\{A, b, c\}$ (non-unique) such that the system

$$
z^{\prime}=A z+y, \quad y=b f(x), \quad x=(c, z) .
$$

is equivalent to equation (1). Here $A$ is a real constant $\ell \times \ell$ matrix; $b$ and $c$ are $\ell$-dimensional vectors; $z \in \mathbb{R}^{\ell}$ is called the state of the system ot the state of equation (1).

Everywhere we suppose that the polynomials $L$ and $M$ are continuous in $\lambda$ and that they are coprime for any $\lambda$. Without loss of generality let $\lambda \in[-1,1]$. Sometimes values of the parameter are called points.

We suppose that the function $f(x, \lambda)$ is continuous in $\{x, \lambda\}$. Let $f(0, \lambda) \equiv 0$, i.e., $x=0$ is an equilibrium of our system, and let $f(x, \lambda)$ be sublinear in $x$ :

$$
\lim _{x \rightarrow 0} \sup _{\lambda \in[-1,1]}\left|x^{-1} f(x, \lambda)\right|=0 .
$$

In this paper we study a Hopf bifurcation, i.e., the existence of small cycles near the zero equilibrium in the state space $\mathbb{R}^{\ell}$ of equation (1).

Definition 1 A value $\lambda_{0}$ of the parameter is called a Hopf bifurcation point or a $\boldsymbol{H B P}$ with the frequency $w_{0}$ for equation (1) if for any sufficiently small $r>0$ there exists a $\lambda_{r}$ such that equation (1) with $\lambda=\lambda_{r}$ has a periodic nonstationary solution $x_{r}=x_{r}(t)$ with the least period $T_{r}$ and also $\lambda_{r} \rightarrow \lambda_{0},\left\|x_{r}\right\|_{C} \rightarrow 0, T_{r} \rightarrow 2 \pi / w_{0}$ as $r \rightarrow 0$.

The use of an auxiliary parameter different from $\lambda$ is rather standard in Hopf bifurcations (see, e.g., [4]). The classical assertion about HBP is the famous Hopf Bifurcation Theorem [4,5]. A Hopf bifurcation with the frequency $w_{0}$ could occur only if $L(p ; \lambda)$ has some roots of the form $n w_{0} i$ for a positive integer $n$; moreover, if there exists exactly one pair of roots of the form $\pm n w_{0} i$, they should be equal to $\pm i w_{0}$. On the other hand, the Hopf Bifurcation Theorem guarantees that $\lambda_{0}$ is a Hopf bifurcation point provided that the polynomial $L(p ; \lambda)$ has the pair of complex roots $\sigma(\lambda) \pm w(\lambda) i, \sigma\left(\lambda_{0}\right)=0, w\left(\lambda_{0}\right)>0$; the numbers $k w\left(\lambda_{0}\right) i$ for $k=0,2,3, \ldots$ are not roots of $L\left(p ; \lambda_{0}\right) ; \sigma^{\prime}\left(\lambda_{0}\right) \neq 0$. Below we formulate a more general statement from [7].

Statement 1 Let for some $\lambda=\lambda_{0}$ the following assumptions be valid:

1. The polynomial $L\left(p ; \lambda_{0}\right)$ has a pair of imaginary roots $\pm w_{0} i\left(w_{0}>0\right)$;

2. $L\left(p ; \lambda_{0}\right)=\left(p^{2}+w_{0}^{2}\right) L_{0}\left(p ; \lambda_{0}\right)$ and $L_{0}\left(p ; \lambda_{0}\right)$ satisfies $L_{0}\left( \pm w_{0} k i ; \lambda_{0}\right) \neq 0$ for any integer $k$; 
3. Due to assumptions 1,2 , the polynomial $L(p ; \lambda)$ has a unique pair of the conjugate roots $\sigma(\lambda) \pm i \tau(\lambda)$ satisfying $\tau\left(\lambda_{0}\right)=w_{0}, \sigma\left(\lambda_{0}\right)=0$ and depending continuously on $\lambda$ in some vicinity of the point $\lambda_{0}$. Let at $\lambda=\lambda_{0}$ the real function $\sigma(\lambda)$ take values of both signs, more precisely, let

$$
\forall \varepsilon>0 \quad \exists \lambda_{-}, \lambda_{+}:\left|\lambda_{ \pm}-\lambda_{0}\right|<\varepsilon, \quad \sigma\left(\lambda_{-}\right) \sigma\left(\lambda_{+}\right)<0 .
$$

Then $\lambda_{0}$ is a HBP with the frequency $w_{0}$ for equation (1).

If there is more information about the roots $\sigma(\lambda) \pm i \tau(\lambda)$ or about the smoothness of $f(x, \lambda)$, then additional statements about the small cycles can be formulated (e.g., statements about continuity and smoothness of the functions $T_{r}, \lambda_{r}$, geometrical properties of the set of cycles $x_{r}$, stability of the cycles etc).

The first condition of Statement 1 is necessary for the point $\lambda_{0}$ to be a HBP. The second and the third conditions are not necessary. The case where the third assumption fails was considered in [8].

Let the second condition of Statement 1 fail:

$$
L\left(p ; \lambda_{0}\right)=\left(p^{2}+w_{0}^{2}\right)\left(p^{2}+n^{2} w_{0}^{2}\right) L_{1}\left(p ; \lambda_{0}\right), \quad L_{1}\left(k w_{0} i, \lambda_{0}\right) \neq 0, \quad k \in \mathbb{Z}
$$

for some unique value of $n=0,1,2, \ldots$ There are 2 different cases: $n=$ $0,1,2,3$ and $n>3$; if $n=0,2,3$ we have strong resonance, if $n>3$ we have weak resonance [5].

According to Statement 1 (if the corresponding conjugate roots cross the imaginary axis), the point $\lambda_{0}$ is a HBP with the frequency $n w_{0}$. We are interested in the following question: is the point $\lambda_{0}$ a HBP with the frequency $\boldsymbol{w}_{0}$ ? Statement 1 is unapplicable to answer the question in the situation considered.

In weak resonance the answer is generically negative $[5,6]$. Only if parameters belong to the so-called "beak of synchronization" the corresponding small cycles exist. The strong resonances have to be considered separately.

In this paper we consider the case of so-called (2:1)-resonance:

$$
L\left(p ; \lambda_{0}\right)=\left(p^{2}+w_{0}^{2}\right)\left(p^{2}+4 w_{0}^{2}\right) L_{1}\left(p ; \lambda_{0}\right)
$$

This case was studied in $[9,10]$ with the approaches of integral manifolds and normal forms for systems of the different type with smooth nonlinearities. Both the approaches allow to analyze phase portraits of the systems.

Here we use another method to study control theory equations (1) with nonsmooth nonlinearities and equations with delays and derivatives. The method is based on harmonic linearization and standard topological methods. 
The main hypothesis is the following:

$$
L(p ; \lambda)=\left(p^{2}+\sigma_{1}(\lambda) p+w_{0}^{2}+\tau_{1}(\lambda)\right)\left(p^{2}+\sigma_{2}(\lambda) p+4 w_{0}^{2}+\tau_{2}(\lambda)\right) L_{1}(p ; \lambda)
$$

where $L_{1}\left( \pm w_{0} k i ; \lambda_{0}\right) \neq 0$ for any integer $k$ and $\sigma_{j}(\lambda), \tau_{j}(\lambda) \rightarrow 0$ as $\lambda \rightarrow \lambda_{0}$, $j=1,2$. We suppose that this hypothesis is valid throughout the paper.

Let us emphasize once more, that if the function $\sigma_{2}(\lambda)$ behaves well, then Statement 1 guarantees that $\lambda_{0}$ is a HBP with the frequency $2 w_{0}$ and the small cycles of the least period $\pi / w$ close to $\pi / w_{0}$ exist. We study the existence of small cycles of the least period $2 \pi / w$ close to $2 \pi / w_{0}$, i.e., the question whether $\lambda_{0}$ is a HBP with the frequency $w_{0}$ or not. The cycles with the least period close to $2 \pi / w_{0}$ are generic 4-dimensional curves (see formula (8) below) in the state space of the control system. Under the assumptions of Statement 1 cycles with the least period close to $\pi / w_{0}$ are almost 2 -dimensional.

Generally speaking, under the conditions of Statement 1 the small cycles exist either for $\lambda>\lambda_{0}$ or for $\lambda<\lambda_{0}$ only. In some degenerate situations (e.g., for Hamiltonian systems) the case where the small cycles exist for $\lambda=\lambda_{0}$ only is natural. The situation where the cycles exist for all $\lambda \neq \lambda_{0}$ sufficiently close to $\lambda_{0}$ is not natural more or less. In (2:1)-resonance case, this last situation is the main case. Moreover, in some general situations, for any $\lambda \neq \lambda_{0}$ there exist two different nontrivial small cycles with periods close to $2 \pi / w_{0}$.

The paper is organized as follows. In the next section we present our main results. There are 2 theorems. One concerns (2:1)-resonance for equations (1) with usual functional nonlinearities. Theorem 2 considers more general nonlinearities with delays and derivatives. Section 3 contains the proof of Theorem 1. In Section 4 we make some remarks, they use notations of Section 3.

Note that to implement our situation, that is to bring two pairs of roots to the imaginary axis simultaneously, we should be able to influence at least two independent parameters of the linear part of the control system.

\section{Main results}

\subsection{Main theorem}

Everywhere we suppose that the continuous functions $\sigma_{j}(\lambda)$ and $\tau_{j}(\lambda)$ in $(2)$ are differentiable at the point $\lambda_{0}$ and denote their derivatives by

$$
\tilde{\alpha}_{j}=\sigma_{j}^{\prime}\left(\lambda_{0}\right), \quad \tilde{\beta}_{j}=\tau_{j}^{\prime}\left(\lambda_{0}\right), \quad j=1,2 .
$$


Define

$$
\alpha_{1}=\frac{\tilde{\alpha}_{1}}{2}, \quad \alpha_{2}=\frac{\tilde{\alpha}_{2}}{4}, \quad \tau=\frac{4 \tilde{\beta}_{1}-\tilde{\beta}_{2}}{16 w_{0}}
$$

and

$$
a+b i=288 w_{0}^{6} \frac{L_{1}\left(i w_{0} ; \lambda_{0}\right) L_{1}\left(2 i w_{0} ; \lambda_{0}\right)}{M\left(i w_{0} ; \lambda_{0}\right) M\left(2 i w_{0} ; \lambda_{0}\right)}
$$

all the numbers $\alpha_{j}, \tau, a, b$ are real. Note that $a+b i \neq 0$.

Theorem 1 Let the nonlinearity in (1) have the form

$$
f(x, \lambda)=x^{2}+\varphi(x, \lambda), \quad \lim _{x \rightarrow 0} \sup _{\lambda \in[-1,1]} \frac{|\varphi(x, \lambda)|}{x^{2}}=0 .
$$

Then the following statements are valid:

1. If $b=0, \alpha_{1}+\alpha_{2} \neq 0$, and $\alpha_{1} \alpha_{2} a>0$, then $\lambda_{0}$ is a HBP with the frequency $w_{0}$ for equation (1). For any $\lambda \neq \lambda_{0}$ sufficiently close to $\lambda_{0}$ there exists at least one small cycle $x(t)$ of the least period $T$ close to $2 \pi / w_{0}$.

2. If $b \neq 0$ and $\alpha_{1} \alpha_{2}>0$, then $\lambda_{0}$ is a HBP with the frequency $w_{0}$ for equation (1). For any $\lambda \neq \lambda_{0}$ sufficiently close to $\lambda_{0}$ there exists at least one small cycle $x(t)$ of the least period $T$ close to $2 \pi / w_{0}$.

3. If $b \neq 0, \alpha_{1} \alpha_{2}<0$,

$$
-4 \alpha_{1} \alpha_{2}\left(a^{2}+b^{2}\right)<\left(a\left(\alpha_{1}-\alpha_{2}\right)+2 b \tau\right)^{2}
$$

and

$$
2 b \tau\left(\alpha_{1}-\alpha_{2}\right)+a\left(\alpha_{1}+\alpha_{2}\right)^{2}<0,
$$

then $\lambda_{0}$ is a HBP with the frequency $w_{0}$ for equation (1). For any $\lambda \neq \lambda_{0}$ sufficiently close to $\lambda_{0}$ there exist at least two different small cycles $x_{1}(t), x_{2}(t)$ with the least periods $T_{1}, T_{2}$, both close to $2 \pi / w_{0}$.

4. If $b \neq 0, \alpha_{1} \alpha_{2}=0, \alpha_{1}^{2}+\alpha_{2}^{2} \neq 0$, and both (6) and (7) hold, then $\lambda_{0}$ is a HBP with the frequency $w_{0}$. For any $\lambda \neq \lambda_{0}$ sufficiently close to $\lambda_{0}$ there exists at least one small cycle $x(t)$ of the least period $T$ close to $2 \pi / w_{0}$.

In statement 4 inequality (6) has a more simple form $a\left(\alpha_{2}-\alpha_{1}\right) \neq 2 b \tau$ (and even more simple, since one $\alpha_{j}$ equals zero). Inequality (7) can be simplified similarly. 
The small periodic solutions $x(t)=x(t ; \lambda)$ considered in Theorem 1 and their periods $T=T(\lambda)$ have the asymptotics

$$
\begin{gathered}
x(t)=\left(\lambda-\lambda_{0}\right)\left(y_{*} \sin w t+q_{*} \sin \left(2 w t+s_{*}\right)\right)+p(t ; \lambda), \\
\|p(t ; \lambda)\|_{C}=o\left(\lambda-\lambda_{0}\right), \\
T=2 \pi / w, \quad w=w_{0}+c_{*}\left(\lambda-\lambda_{0}\right)+o\left(\lambda-\lambda_{0}\right)
\end{gathered}
$$

where $y_{*} \neq 0, \lambda \neq \lambda_{0}$. Evidently, the shift of time in (8) generates the continuum of solutions $x(t+s)$, all of them have the same cyclic trajectory. Under the conditions of statement 3 , there are at least two branches of periodic solutions with the asymptotics (8), (9), the coefficients $y_{*}, q_{*}, s_{*}, c_{*}$ are different for these branches.

If $\alpha_{1} \alpha_{2}<0$ and the inverse inequality is valid either instead of (6) or instead of (7), then system (1) does not have periodic solutions (8), (9). If either in (6) or in (7) there is the equality instead of the inequality, then the existence of such solutions may be defined by higher order terms of the expansion of the nonlinearity.

If the term $\varphi(x, \lambda)$ in (5) satisfies the Lipschitz condition

$$
\left|\varphi\left(x_{1}, \lambda\right)-\varphi\left(x_{2}, \lambda\right)\right| \leq c(\rho)\left|x_{1}-x_{2}\right|, \quad\left|x_{1}\right|,\left|x_{2}\right| \leq \rho, \quad|\lambda| \leq 1
$$

where $c(\rho) / \rho$ vanishes as $\rho \rightarrow 0$, then Theorem 1 gives the exact number of small periodic solutions (8) with periods (9). Under the assumptions of statements 1, 2, and 4 there is a unique branch of such solutions, under the assumptions of statement 3 there are exactly two branches.

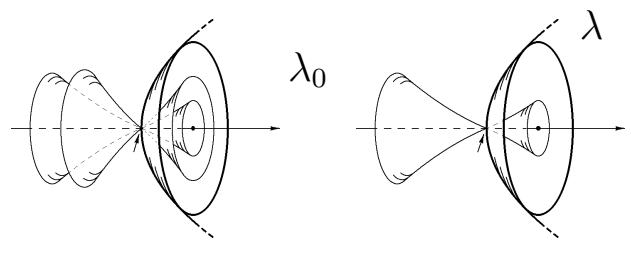

Fig. 1a. Statement 3
Fig. 1b. Statements 1,2,4

Fig.1. Various statements of Theorem 1 


\subsection{Bifurcation diagrams}

Theorem 1 is illustrated with Fig. 1 - Fig. 3.

Fig. 1 presents local pictures in the space $\{z, \lambda\}$ ( $z$ is a point of the state space). The "cup" of almost $\pi / w_{0}$-periodic cycles (drown with thick lines) is accompanied by different numbers of the "cones" of almost $2 \pi / w_{0}$-periodic cycles (thin lines). The almost $\pi / w_{0}$-periodic cycles are really close to circles. The almost $2 \pi / w_{0}$-periodic cycles are close to general 4 -dimensional curves, they are drawn as circles only conditionally.

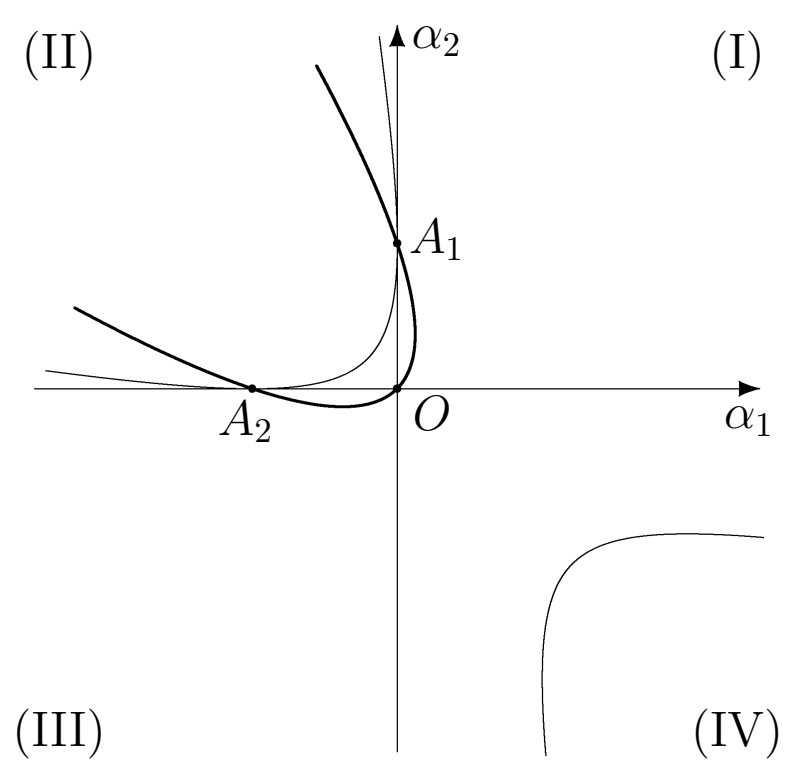

Fig. 2. Inequalities (6) and (7)

On Fig. 2, 3 we show bifurcation diagrams on the plane $\left\{\alpha_{1}, \alpha_{2}\right\}$ for fixed $a, b, \tau$. In different parts of the plane there are different numbers of small cycles with the least periods close to $2 \pi / w_{0}$. We suppose that $a, b, \tau$ are nonzero. The values of $\lambda$ are close to $\lambda_{0}$.

Fig. 2 illustrates statement 3 of Theorem 1.

Relation (6) with the equality instead of the inequality defines a hyperbola with the branches in the (II)-nd and the (VI)-th quadrants. One branch is tangent to the coordinate axes at the points $A_{1}=\{0,2 b \tau / a\}$ and $A_{2}=$ $\{-2 b \tau / a, 0\}$, another branch does not intersect the axes. Relation (7) with the equality instead of the inequality defines the parabola that passes through the origin $O$ and intersects the hyperbola at the same points $A_{1}$ and $A_{2}$. Fig. 2 
is symmetric with respect to the line $\alpha_{1}+\alpha_{2}=0$. The second symmetry axis of the hyperbola is $\alpha_{2}-\alpha_{1}=-2 a \tau / b$.

Inequality (6) is valid between the branches of the hyperbola, i.e., in the connected part of the plane including the origin. Inequality (7) is valid inside the parabola if $a>0$ and outside the parabola if $a<0$. If $a>0$, the conditions of statement 3 are satisfied inside the curvilinear triangle $A_{1} O A_{2}$, its boundary consists of the segments $O A_{1}, O A_{2}$ and the arc $A_{1} A_{2}$ of the hyperbola. If $a<0$, the conditions of statement 3 are satisfied in the three unbounded domains. On Fig. 2 one domain is the part of the (IV)-th quadrant between the corresponding branch of the hyperbola and the coordinate halfaxes. The other two domains are the "beaks" with the vertices $A_{1}$ and $A_{2}$ in the (II)-nd quadrant, the boundary of each beak consists of the coordinate half-axis and the part of the other branch of the hyperbola.

Fig. 3 shows, how the number of branches of almost $2 \pi / w_{0}$-periodic cycles depends on $\alpha_{1}$ and $\alpha_{2}$. The domains with different numbers of branches are separated by the parts of the coordinate axes and the hyperbola.

Fig. 2, 3 are drawn for the case $a b \tau>0$. If $a b \tau<0$, the pictures are symmetric to these figures with respect to the line $\alpha_{1}=\alpha_{2}$.

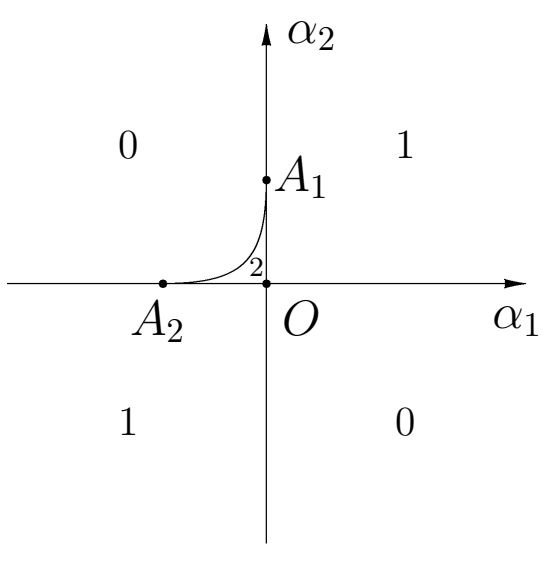

Fig. 3a. The case $a>0$

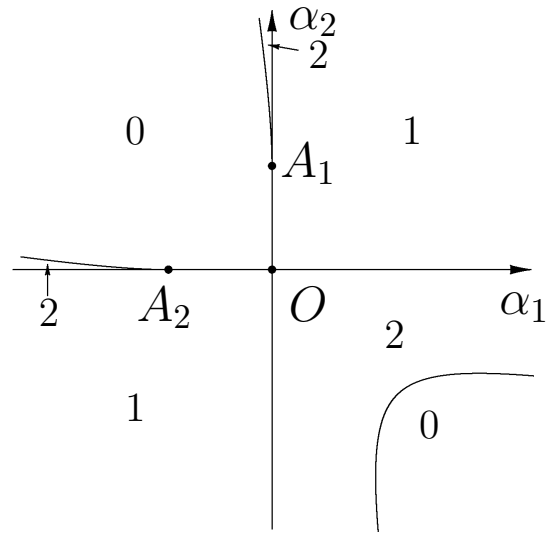

Fig. 3b. The case $a<0$

Fig. 3. Bifurcation diagrams

Let $b=0$. For example, it takes place if $L$ is a 4 th order polynomial and $M \equiv 1$. In this case, relations (6), (7) are equivalent to $\alpha_{1}+\alpha_{2} \neq 0, a<0$. If $\alpha_{1} \alpha_{2}<0$, this coincides with the conditions of statement 1 . 


\subsection{Nonlinearity with delays and derivatives}

Consider the equation

$$
L\left(\frac{d}{d t} ; \lambda\right) x=M\left(\frac{d}{d t} ; \lambda\right) f\left(x(t), x(t-\theta), x^{\prime}(t), \lambda\right),
$$

more general than (1). Here the degrees of the polynomials satisfy $\ell>m+1$.

Theorem 2 Let the nonlinearity $f(x, y, z, \lambda)$ in (10) have the form

$$
d_{1}(\lambda) x^{2}+d_{2}(\lambda) y^{2}+d_{3}(\lambda) z^{2}+d_{4}(\lambda) x y+d_{5}(\lambda) x z+d_{6}(\lambda) y z+F(x, y, z, \lambda)
$$

(the quadratic part is of the most general type) with

$$
\lim _{|x|+|y|+|z| \rightarrow 0} \sup _{\lambda \in[-1,1]} \frac{|F(x, y, z, \lambda)|}{(|x|+|y|+|z|)^{2}}=0 .
$$

Define the numbers $\alpha_{j}$ and $\tau$ by formulas (3) and the numbers $a, b$ by the new formula (different from (4))

$$
a+b i=-\frac{144 w_{0}^{6}}{A\left(\lambda_{0}\right) B\left(\lambda_{0}\right)} \frac{L_{1}\left(i w_{0} ; \lambda_{0}\right) L_{1}\left(2 i w_{0} ; \lambda_{0}\right)}{M\left(i w_{0} ; \lambda_{0}\right) M\left(2 i w_{0} ; \lambda_{0}\right)}
$$

where

$$
\begin{aligned}
A(\lambda) & =d_{1}(\lambda)+d_{2}(\lambda) e^{-i \theta w_{0}}+2 d_{3}(\lambda) w_{0}^{2} \\
& +\frac{d_{4}(\lambda)}{2}\left(e^{i \theta w_{0}}+e^{-2 i \theta w_{0}}\right)+\frac{i}{2} d_{5}(\lambda) w_{0}+i d_{6}(\lambda) w_{0}\left(e^{i \theta w_{0}}-\frac{1}{2} e^{-2 i \theta w_{0}}\right), \\
B(\lambda) & =-\frac{1}{2}\left(d_{1}(\lambda)+d_{2}(\lambda) e^{-2 i \theta w_{0}}-d_{3}(\lambda) w_{0}^{2}+d_{4}(\lambda) e^{-i \theta w_{0}}\right. \\
& \left.+i d_{5}(\lambda) w_{0}+i d_{6}(\lambda) w_{0} e^{-i \theta w_{0}}\right) .
\end{aligned}
$$

Then all the statements 1 - 4 of Theorem 1 are valid for equation (10).

In the formulation of Theorem 2 we suppose that $A\left(\lambda_{0}\right) \neq 0$ and $B\left(\lambda_{0}\right) \neq 0$.

The proof of this theorem is almost the same as the proof of Theorem 1. We have only to take care of the correct choice of functional spaces.

Of course, it is possible to consider nonlinearities with many delays and with higher derivatives, derivatives of terms with delays, etc. One can easily change correspondingly the formulation of Theorem 2 . 


\section{Proof of Theorem 1}

\subsection{General scheme}

Consider the equation

$$
L\left(w \frac{d}{d t} ; \lambda\right) x=M\left(w \frac{d}{d t} ; \lambda\right) f(x, \lambda)
$$

with the additional parameter $w$, its values are close to $w_{0}$. Evidently, every $2 \pi$-periodic solution $x(t)$ of equation (11) defines the $2 \pi / w$-periodic solution $x(w t)$ of equation (1). We identify $2 \pi$-periodic functions with their restrictions to the segment $[0,2 \pi]$. Let us set

$$
P x(t)=\frac{1}{\pi} \int_{0}^{2 \pi} \cos (t-s) x(s) d s+\frac{1}{\pi} \int_{0}^{2 \pi} \cos 2(t-s) x(s) d s, \quad Q x=x-P x
$$

and split equation (11) into the system ${ }^{4}$

$$
\begin{aligned}
& L\left(w \frac{d}{d t} ; \lambda\right) \operatorname{Px}(t)=M\left(w \frac{d}{d t} ; \lambda\right) \operatorname{Pf}(x(t), \lambda), \\
& L\left(w \frac{d}{d t} ; \lambda\right) Q x(t)=M\left(w \frac{d}{d t} ; \lambda\right) Q f(x(t), \lambda) .
\end{aligned}
$$

We look for $2 \pi$-periodic solutions of this system of the form

$$
x(t)=r \sin t+\xi \sin 2 t+\eta \cos 2 t+h(t), \quad r>0
$$

where $h(t)=Q x(t)$. Note that every such solution is included in the continuum of shifted solutions $x(t+s), 0 \leq s<2 \pi$, all of them have the same cyclic trajectory. Only one solution of this continuum has the form (14) with $r>0$.

Below we show that for small solutions (14) the norm of $h$ is of smaller order than the norm of $P x$, namely,

$$
\|h\|_{C} \leq \kappa_{0} \rho^{2}, \quad \rho^{2}=r^{2}+\xi^{2}+\eta^{2},
$$

with some constant $\kappa_{0}>0$ independent of $\lambda$ and $w$. Since $f(x, \lambda)=x^{2}+o\left(x^{2}\right)$ and $\|h\|_{C}=O\left(\rho^{2}\right)$, it follows that

$$
f(x(t), \lambda)=-r \eta \sin t+r \xi \cos t-\frac{r^{2}}{2} \cos 2 t+h_{1}(t)+h_{2}(t)
$$

where $h_{1}(t)=Q f(x(t), \lambda)$ and $\left\|h_{2}\right\|_{C}=o\left(\rho^{2}\right)$ as $\rho \rightarrow 0$.

$\overline{4}$ The projectors $P$ and $Q$ commute with the operator of differentiation. 
Our equations and their solutions are considered in spaces of real-valued functions defined on the segment $[0,2 \pi]$. For simplicity, we use notations of complex analysis, every algebraic equation in $\mathbb{C}$ denotes a pair of real equations. If we write a vector field with a complex component, this is just the denomination for the two real components. We stress that all derivatives are real.

Consider equation (12). Let us multiply it by $e^{-i t}$ and integrate over the segment $[0,2 \pi]$. Using the identity

$$
\int_{0}^{2 \pi} e^{-i n t} \frac{d^{k}}{d t^{k}} P x(t) d t=(i n)^{k} \int_{0}^{2 \pi} e^{-i n t} P x(t) d t
$$

and expressions (14) and (16) for $x(t)$ and $f(x(t), \lambda)$, we obtain

$$
-i r L(i w ; \lambda)=r M(i w ; \lambda)(\xi+i \eta)+\psi_{1}, \quad \psi_{1}=o\left(\rho^{2}\right) .
$$

Similarly, we multiply this equation by $e^{-2 i t}$, integrate over $[0,2 \pi]$, and get

$$
(\xi+i \eta) L(2 i w ; \lambda)=-i \frac{r^{2}}{2} M(2 i w ; \lambda)+\psi_{2}, \quad \psi_{2}=o\left(\rho^{2}\right) .
$$

System (17), (18) is equivalent to (12).

To explain our results, we omit for the moment small terms in (17) and (18). First, we omit the terms $\psi_{i}$ and rewrite the system as

$$
-i L(i w ; \lambda)=M(i w ; \lambda)(\xi+i \eta), \quad(\xi+i \eta) L(2 i w ; \lambda)=-i \frac{r^{2}}{2} M(2 i w ; \lambda) .
$$

Equivalently,

$$
\xi+i \eta=-i \frac{L(i w ; \lambda)}{M(i w ; \lambda)}, \quad r^{2}=2 \frac{L(i w ; \lambda) L(2 i w ; \lambda)}{M(i w ; \lambda) M(2 i w ; \lambda)} .
$$

Secondly, we linearize ${ }^{5}$ the rational functions $W_{1}=L(i w ; \lambda) / M(i w ; \lambda)$ and $W_{2}=L(2 i w ; \lambda) / M(2 i w ; \lambda)$ at the point $\lambda=\lambda_{0}, w=w_{0}$ :

$$
\begin{aligned}
& W_{1}=3 w_{0}^{2} \frac{L_{1}\left(i w_{0} ; \lambda_{0}\right)}{M\left(i w_{0} ; \lambda_{0}\right)}\left(-2 w_{0}\left(w-w_{0}\right)+\left(\tilde{\beta}_{1}+i w_{0} \tilde{\alpha}_{1}\right)\left(\lambda-\lambda_{0}\right)\right)+\phi_{1} \\
& W_{2}=-3 w_{0}^{2} \frac{L_{1}\left(2 i w_{0} ; \lambda_{0}\right)}{M\left(2 i w_{0} ; \lambda_{0}\right)}\left(-8 w_{0}\left(w-w_{0}\right)+\left(\tilde{\beta}_{2}+2 i w_{0} \tilde{\alpha}_{2}\right)\left(\lambda-\lambda_{0}\right)\right)+\phi_{2}
\end{aligned}
$$

and retain only the main linear parts of these expressions. Here the small terms satisfy $\phi_{j}=o\left(\left|\lambda-\lambda_{0}\right|+\left|w-w_{0}\right|\right)$ as $\lambda \rightarrow \lambda_{0}, w \rightarrow w_{0}$; omitting these

5 We would like to emphasize that this linearization is valid without any smoothness in $\lambda$ of the coefficients of the polynomials $L_{1}$ and $M$. 
terms, we replace system (20) by the close system

$$
\begin{aligned}
\xi+i \eta= & -6 i w_{0}^{3} \frac{L_{1}\left(i w_{0} ; \lambda_{0}\right)}{M\left(i w_{0} ; \lambda_{0}\right)}\left(w_{0}-w+\left(\frac{\tilde{\beta}_{1}}{2 w_{0}}+i \frac{\tilde{\alpha}_{1}}{2}\right)\left(\lambda-\lambda_{0}\right)\right), \\
r^{2}= & -288 w_{0}^{6} \frac{L_{1}\left(i w_{0} ; \lambda_{0}\right) L_{1}\left(2 i w_{0} ; \lambda_{0}\right)}{M\left(i w_{0} ; \lambda_{0}\right) M\left(2 i w_{0} ; \lambda_{0}\right)}\left(w_{0}-w+\left(\frac{\tilde{\beta}_{1}}{2 w_{0}}+i \frac{\tilde{\alpha}_{1}}{2}\right)\left(\lambda-\lambda_{0}\right)\right) \\
& \times\left(w_{0}-w+\left(\frac{\tilde{\beta}_{2}}{8 w_{0}}+i \frac{\tilde{\alpha}_{2}}{4}\right)\left(\lambda-\lambda_{0}\right)\right) .
\end{aligned}
$$

With the notation (3), (4), and

$$
c+d i=6 i w_{0}^{3} \frac{L_{1}\left(i w_{0} ; \lambda_{0}\right)}{M\left(i w_{0} ; \lambda_{0}\right)}, \quad \beta=-\frac{4 \tilde{\beta}_{1}+\tilde{\beta}_{2}}{16 w_{0}},
$$

this system takes the form

$$
\begin{aligned}
& \xi+i \eta=-(c+d i)\left(w_{0}-w+\left(\alpha_{1} i+\tau-\beta\right)\left(\lambda-\lambda_{0}\right)\right) \\
& r^{2}=-(a+b i)\left(w_{0}-w+\left(\alpha_{1} i+\tau-\beta\right)\left(\lambda-\lambda_{0}\right)\right)\left(w_{0}-w+\left(\alpha_{2} i-\tau-\beta\right)\left(\lambda-\lambda_{0}\right)\right) .
\end{aligned}
$$

Define the new variables $z, y, u, v$ by

$$
w-w_{0}=(z-\beta)\left(\lambda-\lambda_{0}\right), r=y\left(\lambda-\lambda_{0}\right), \quad \xi=u\left(\lambda-\lambda_{0}\right), \eta=v\left(\lambda-\lambda_{0}\right)
$$

and rewrite the system as

$$
\begin{aligned}
u+v i & =(c+d i)\left(z-\tau-\alpha_{1} i\right), \\
-y^{2} & =(a+b i)\left(z-\tau-\alpha_{1} i\right)\left(z+\tau-\alpha_{2} i\right) .
\end{aligned}
$$

Here equation (25) plays the main role: if its solutions $z, y$ are found, then $u, v$ are determined explicitly by (24). We stress again that all the variables and unknowns are real.

Equation (25) is studied in the next subsection. In the rest of the proof, we show that every pair of its regular solutions (precise definitions are given below) determines a small-amplitude $2 \pi$-periodic solution (14) of equation (11).

\subsection{Quadratic equation}

Consider the real and the complex components of equation (25) separately:

$$
\begin{aligned}
y^{2}+a\left(z^{2}-\tau^{2}-\alpha_{1} \alpha_{2}\right)+b\left(z\left(\alpha_{1}+\alpha_{2}\right)+\tau\left(\alpha_{1}-\alpha_{2}\right)\right) & =0, \\
b\left(z^{2}-\tau^{2}-\alpha_{1} \alpha_{2}\right)-a\left(z\left(\alpha_{1}+\alpha_{2}\right)+\tau\left(\alpha_{1}-\alpha_{2}\right)\right) & =0 .
\end{aligned}
$$


Let us linearize the left-hand side of this system at a point $(z, y)$ and consider the corresponding Jacobian

$$
J(z, y)=\left|\begin{array}{rr}
2 a z+b\left(\alpha_{1}+\alpha_{2}\right) & 2 y \\
2 b z-a\left(\alpha_{1}+\alpha_{2}\right) & 0
\end{array}\right| .
$$

A solution $\left(z_{*}, y_{*}\right)$ of equation (25) (equivalently, of system $\left.(26),(27)\right)$ is called regular if $J\left(z_{*}, y_{*}\right) \neq 0$, i.e., $y_{*} \neq 0$ and

$$
2 b z_{*} \neq a\left(\alpha_{1}+\alpha_{2}\right) \text {. }
$$

Evidently, if $\left(z_{*}, y_{*}\right)$ is regular, $\left(z_{*},-y_{*}\right)$ is a regular solution too. Thus, every root $z_{*}$ of equation (27) such that relations (29) and

$$
a\left(z_{*}^{2}-\tau^{2}-\alpha_{1} \alpha_{2}\right)+b\left(z_{*}\left(\alpha_{1}+\alpha_{2}\right)+\tau\left(\alpha_{1}-\alpha_{2}\right)\right)<0
$$

are valid defines the pair $\left(z_{*}, \pm y_{*}\right)$ of regular solutions of equation $(25)$.

Lemma 1 The following statements are valid:

1. If $b=0, \alpha_{1}+\alpha_{2} \neq 0$, and $\alpha_{1} \alpha_{2} a>0$, then there exists a pair of regular solutions $\left(z_{*}, \pm y_{*}\right)$ of $(25)$.

2. If $b \neq 0$ and $\alpha_{1} \alpha_{2}>0$, then there exists a pair of regular solutions of (25).

3. If $b \neq 0, \alpha_{1} \alpha_{2}<0$, and inequalities (6) and (7) are valid, then there exist two different pairs of regular solutions of (25).

4. If $b \neq 0, \alpha_{1} \alpha_{2}=0, \alpha_{1}^{2}+\alpha_{2}^{2} \neq 0$, and (6) and (7) are valid, then there exists a pair of regular solutions of (25).

If the parameters $a, b, \alpha_{j}, \tau$ do not satisfy conditions of some part of Lemma 1 , then either equation (25) has no solutions at all or it has some solutions, but not regular. For example, there are no regular solutions if $b=\alpha_{1}+\alpha_{2}=0$ and in some other cases.

Proof. To prove the lemma, we should determine the number of the roots of equation (27) satisfying (29), (30).

Let $b=0, \alpha_{1}+\alpha_{2} \neq 0$. Since $a+b i \neq 0$, also $a \neq 0$. Therefore equation (27) has the form $z\left(\alpha_{1}+\alpha_{2}\right)+\tau\left(\alpha_{1}-\alpha_{2}\right)=0$, i.e., it has a unique root

$$
z^{\star}=\tau \frac{\alpha_{2}-\alpha_{1}}{\alpha_{1}+\alpha_{2}}
$$


Inequality (29) obviously holds. Substituting (31) in (30), we obtain

$$
a\left(\tau^{2}\left(\left(\frac{\alpha_{2}-\alpha_{1}}{\alpha_{1}+\alpha_{2}}\right)^{2}-1\right)-\alpha_{1} \alpha_{2}\right)=-\alpha_{1} \alpha_{2} a\left(\frac{4 \tau^{2}}{\left(\alpha_{1}+\alpha_{2}\right)^{2}}+1\right)<0,
$$

which is true whenever $\alpha_{1} \alpha_{2} a>0$. This proves statement 1 .

Below in the proof we suppose $b \neq 0$. Therefore $(27)$ is a quadratic equation with the discriminant

$$
\begin{aligned}
D & =a^{2}\left(\alpha_{1}+\alpha_{2}\right)^{2}+4 \tau^{2} b^{2}+4 \alpha_{1} \alpha_{2} b^{2}+4 a b \tau\left(\alpha_{1}-\alpha_{2}\right) \\
& =\left(a\left(\alpha_{1}-\alpha_{2}\right)+2 \tau b\right)^{2}+4 \alpha_{1} \alpha_{2}\left(a^{2}+b^{2}\right) .
\end{aligned}
$$

First note that $D$ is positive iff either $\alpha_{1} \alpha_{2}>0$ or estimates $\alpha_{1} \alpha_{2} \leq 0$ and (6) are valid. Therefore equation (27) has two different roots under the assumptions of each of statements $2-4$. Secondly, condition (29) is equivalent to $D \neq 0$, hence it is valid for both the roots and we should check (30) only.

Since the roots satisfy

$$
z_{*}^{2}-\tau^{2}-\alpha_{1} \alpha_{2}=\left(z_{*}\left(\alpha_{1}+\alpha_{2}\right)+\tau\left(\alpha_{1}-\alpha_{2}\right)\right) a / b,
$$

estimate (30) is equivalent to

$$
b\left(a^{2} / b^{2}+1\right)\left(z_{*}\left(\alpha_{1}+\alpha_{2}\right)+\tau\left(\alpha_{1}-\alpha_{2}\right)\right)<0 .
$$

If $\alpha_{1}+\alpha_{2}=0$ (this can be the case under the conditions of statement 3 only), this estimate has the form $b \tau\left(\alpha_{1}-\alpha_{2}\right)<0$, i.e., it coincides with (7). Therefore each of the roots defines the pair of regular solutions of equation (25).

Suppose $\alpha_{1}+\alpha_{2} \neq 0$. Then number (31) is defined and we can rewrite (32) as

$$
b\left(\alpha_{1}+\alpha_{2}\right)\left(z_{*}-z^{\star}\right)<0 .
$$

Consider the polynomial

$$
G(z)=z^{2}-\tau^{2}-\alpha_{1} \alpha_{2}-\left(z\left(\alpha_{1}+\alpha_{2}\right)+\tau\left(\alpha_{1}-\alpha_{2}\right)\right) a / b .
$$

Its value at the point $z^{\star}$ equals

$$
G\left(z^{\star}\right)=\tau^{2}\left(\left(\frac{\alpha_{2}-\alpha_{1}}{\alpha_{1}+\alpha_{2}}\right)^{2}-1\right)-\alpha_{1} \alpha_{2}=-\alpha_{1} \alpha_{2}\left(\frac{4 \tau^{2}}{\left(\alpha_{1}+\alpha_{2}\right)^{2}}+1\right) .
$$

If $\alpha_{1} \alpha_{2}>0$, then $G\left(z^{\star}\right)<0$. This means that the point $z^{\star}$ lies between the roots of $G(\cdot)$, i.e., of equation (27). Therefore (33) is satisfied exactly for one of the roots and this is equivalent to statement 2 . 
If $\alpha_{1} \alpha_{2}=0$, then $z^{\star}$ is a root of $G(\cdot)$, it does not satisfy (33). By the Vieta theorem, the other root is $z_{*}=\left(\alpha_{1}+\alpha_{2}\right) a / b-z^{\star}$. Substituting this in (33), we obtain

$$
\left(\alpha_{1}+\alpha_{2}\right)\left(\left(\alpha_{1}+\alpha_{2}\right) a-2 b z^{\star}\right)<0,
$$

which is equivalent to condition (7). This proves statement 4 .

If $\alpha_{1} \alpha_{2}<0$, then $G\left(z^{\star}\right)>0$. This means that either both the roots satisfy (33) or both do not. Moreover, they satisfy (33) iff their half-sum $\left(\alpha_{1}+\alpha_{2}\right) a /(2 b)$ satisfies (33), i.e., (34) is valid. Therefore condition (7) implies statement 3 and the proof is complete.

\subsection{Equivalent system}

In this subsection, we pass from (12), (13) to the equivalent system, which is then studied by topological methods. Equation (13) is equivalent to the operator equation

$$
h(t)=H(w, \lambda) Q f(x(t), \lambda)
$$

where $h=Q x$. Here $H=H(w, \lambda)$ denotes the linear operator that maps a function $\nu$ to a $2 \pi$-periodic solution $\chi=H(w, \lambda) \nu$ of the equation

$$
L\left(w \frac{d}{d t} ; \lambda\right) \chi=M\left(w \frac{d}{d t} ; \lambda\right) \nu
$$

This operator is well defined in the subspace $\mathbb{E}=Q L^{2} \subset L^{2}$. It is completely continuous and normal, its eigenvalues are $\mu_{k}(w, \lambda)=M(w k i ; \lambda) / L(w k i ; \lambda)$, $k=0, \pm 3, \pm 4, \ldots$ The norms of the operators $H(w, \lambda)$ are uniformly bounded:

$$
\sup _{w, \lambda}\|H(w, \lambda)\|_{\mathbb{E} \rightarrow \mathbb{E}}=\sup _{w, \lambda}\left|\mu_{k}(w, \lambda)\right| \leq \kappa_{1} .
$$

Here we take supremum with respect to all $w$ sufficiently close to $w_{0}$ and $\lambda$ sufficiently close to $\lambda_{0}$. "Sufficiently close" means that for these $w$ and $\lambda$ the polynomial $L(p ; \lambda)$ has no roots $\pm w k i$ with integer $k \neq 1,2$. It is possible to consider such $w$ and $\lambda$ due to our assumption that the polynomial $L(p ; \lambda)$ has the form (2) and depends continuously on $\lambda$.

Moreover, the operator $H(w, \lambda)$ with fixed $w, \lambda$ acts from the space $\mathbb{E}$ to the subspace $\mathbb{E}_{c}=\{\chi \in \mathbb{E} \cap C, \chi(0)=\chi(2 \pi)\} \subset C$ and

$$
\sup _{w, \lambda}\|H(w, \lambda)\|_{\mathbb{E} \rightarrow C} \leq \kappa_{2} .
$$

If $w$ and $\lambda$ range over sufficiently small vicinities $U_{1}, U_{2}$ of the points $w_{0}$ and $\lambda_{0}$, then the operator $H(w, \lambda) \nu$ acting from the product $U_{1} \times U_{2} \times \mathbb{E}$ to $\mathbb{E}_{c}$ is completely continuous with respect to the set of its arguments $w, \lambda$, and $\nu$. 
Now, it is easy to obtain a priori estimate (15) for all sufficiently small solutions (14) of system (12), (13). Indeed, (35) implies

$$
\begin{aligned}
\|h\|_{C} & \leq\|H(w, \lambda)\|_{\mathbb{E} \rightarrow C}\|Q f(x(t), \lambda)\|_{L^{2}} \leq \kappa_{2}\|f(x(t), \lambda)\|_{L^{2}} \\
& \leq \kappa_{2} \sqrt{2 \pi}\|f(x(t), \lambda)\|_{C},
\end{aligned}
$$

hence $f(x, \lambda)=x^{2}+o\left(x^{2}\right)$ implies the estimate $\|h\|_{C} \leq \kappa_{3}\|x\|_{C}^{2}$ with some common $\kappa_{3}$ for all $w \in U_{1}, \lambda \in U_{2}$. Since

$$
\rho \leq \sqrt{2}\|x\|_{C}, \quad\|h\|_{C} \leq\|x\|_{C}+3 \rho, \quad\|x\|_{C} \leq\|h\|_{C}+3 \rho,
$$

the amplitude $\|x\|_{C}$ is small iff both $\|h\|_{C}$ and $\rho$ are small, therefore (15) follows from

$$
\|h\|_{C} \leq \kappa_{3}\|x\|_{C}^{2} \leq \kappa_{3}\left(\|h\|_{C}+3 \rho\right)^{2} .
$$

As we have seen in Subsection 3.1, estimate (15) implies that equation (12) is equivalent to system (17), (18) with $\psi_{j}=o\left(\rho^{2}\right), \rho \rightarrow 0$. Suppose $\lambda \neq \lambda_{0}$. Changing the variables in (17), (18) by formulas (23), we obtain

$$
\frac{-i y L(i w ; \lambda)}{\left(\lambda-\lambda_{0}\right) M(i w ; \lambda)}=y(u+v i)+\varphi_{1}, \frac{(u+v i) L(2 i w ; \lambda)}{\left(\lambda-\lambda_{0}\right) M(2 i w ; \lambda)}=-i \frac{y^{2}}{2}+\varphi_{2}
$$

where $\varphi_{j} \rightarrow 0$ as $\lambda \rightarrow \lambda_{0}$ and this convergence is uniform with respect to the variables $y, u, v, z$ if they are taken from any bounded domain of $\mathbb{R}^{4}$. Furthermore, with the notation (3), (4), and (22), formulas (21) take the form

$$
\begin{aligned}
\frac{L(i w ; \lambda)}{\left(\lambda-\lambda_{0}\right) M(i w ; \lambda)} & =i(c+d i)\left(z-\tau-\alpha_{1} i\right)+\varphi_{3}, \\
\frac{L(2 i w ; \lambda)}{\left(\lambda-\lambda_{0}\right) M(2 i w ; \lambda)} & =\frac{i(a+b i)}{2(c+d i)}\left(z+\tau-\alpha_{2} i\right)+\varphi_{4},
\end{aligned}
$$

therefore we can rewrite system (38) as

$y(c+d i)\left(z-\tau-\alpha_{1} i\right)=y(u+v i)+\varphi_{5}, \quad \frac{a+b i}{c+d i}(u+v i)\left(z+\tau-\alpha_{2} i\right)=-y^{2}+\varphi_{6}$

with $\varphi_{j}$ vanishing uniformly as above. Finally, for $y \neq 0$ this system is equivalent to

$$
\begin{aligned}
u+v i & =(c+d i)\left(z-\tau-\alpha_{1} i\right)+\varphi_{7}, \\
-y^{2} & =(a+b i)\left(z-\tau-\alpha_{1} i\right)\left(z+\tau-\alpha_{2} i\right)+\varphi_{8}
\end{aligned}
$$

where $\varphi_{7}, \varphi_{8}$ vanish uniformly with respect to $y, u, v, z$ whenever the bounded range of these variables in $\mathbb{R}^{4}$ is closed and does not intersect the hyperplane $y=0$; (note that system (24), (25) is the main part of system (39), (40)).

This completes the reduction of equation (11) to the system of equations (35), (39), and (40) with the unknowns $y, u, v, z$, and $h=h(t)$; formulas (14) 
and (23) relate the $2 \pi$-periodic solutions $x(t)$ of (11) with the solutions of this system. Recall that we look for solutions (14) with $r>0$, which is $y\left(\lambda-\lambda_{0}\right)>0$ in other terms.

\subsection{Homotopy}

For any $\lambda$ close to $\lambda_{0}, \lambda \neq \lambda_{0}$, define the vector field

$$
\Phi(u, v, z, y, h)=\left(\begin{array}{c}
u+v i-(c+d i)\left(z-\tau-\alpha_{1} i\right)-\varphi_{7} \\
y^{2}+(a+b i)\left(z-\tau-\alpha_{1} i\right)\left(z+\tau-\alpha_{2} i\right)+\varphi_{8} \\
h-H(w, \lambda) Q f(x(t), \lambda)
\end{array}\right)
$$

in the space $\mathbb{R}^{4} \times \mathbb{E}_{c}$. By construction, zeros of $\Phi$ are solutions of system (35), (39), (40). To prove the existence of at least one zero, we show that the rotation (see [11]) of the completely continuous vector field $\Phi$ on the boundary of an appropriate domain is nonzero.

Let $\left(z_{*}, y_{*}\right)$ be a regular solution of equation (25). Set

$$
u_{*}+v_{*} i=(c+d i)\left(z_{*}-\tau-\alpha_{1} i\right) .
$$

Denote by $\Psi=\Theta \oplus I$ the direct sum of the four-dimensional vector field

$$
\Theta(u, v, z, y)=\left(\begin{array}{c}
u+v i-(c+d i)\left(z-\tau-\alpha_{1} i\right) \\
y^{2}+(a+b i)\left(z-\tau-\alpha_{1} i\right)\left(z+\tau-\alpha_{2} i\right)
\end{array}\right)
$$

with the identity vector field $I h=h\left(h \in \mathbb{E}_{c}\right)$, and by $\Omega=\Omega(R, \varepsilon, \lambda)$ the direct product of the domains

$$
\begin{aligned}
& \Omega_{1}=\Omega_{1}(\varepsilon)=\left\{\zeta \in \mathbb{R}^{4}:\left|\zeta-\zeta_{*}\right|<\varepsilon\right\}, \\
& \Omega_{2}=\Omega_{2}(R, \lambda)=\left\{h \in \mathbb{E}_{c}:\|h\|_{C}<R\left(\lambda-\lambda_{0}\right)^{2}\right\}
\end{aligned}
$$

where $\zeta=(u, v, z, y), \zeta_{*}=\left(u_{*}, v_{*}, z_{*}, y_{*}\right)$, and $|\zeta|=\max \{|u|,|v|,|z|,|y|\}$. Consider the linear deformation $\mu \Phi+(1-\mu) \Psi, \mu \in[0,1]$, connecting the vector fields $\Phi$ and $\Psi$. We now choose the parameters $R>0$ and $\varepsilon \in(0,1)$ such that this deformation is nondegenerate, i.e., $\mu \Phi+(1-\mu) \Psi \neq 0$ on the boundary $\partial \Omega$ of the domain $\Omega$ for all sufficiently small $\left|\lambda-\lambda_{0}\right|>0$. A nondegenerate deformation is also called a homotopy.

The boundary $\partial \Omega$ is the join of the sets $\partial \Omega_{1} \times \bar{\Omega}_{2}$ and $\bar{\Omega}_{1} \times \partial \Omega_{2}$ where $\bar{\Omega}_{j}$ and $\partial \Omega_{j}$ are the closure and the boundary of $\Omega_{j}$. Suppose that the $h$ component of the field $\mu \Phi+(1-\mu) \Psi$ is degenerate at some point of $\partial \Omega$, 
i.e., $h=\mu H(w, \lambda) Q f(x(t), \lambda)$. Like in Subsection 3.3, this relation implies estimates (36), (37), and hence (15) is valid:

$$
\|h\|_{C} \leq \kappa_{0} \rho^{2}=\kappa_{0}\left(\xi^{2}+\eta^{2}+r^{2}\right)=\kappa_{0}\left(u^{2}+v^{2}+y^{2}\right)\left(\lambda-\lambda_{0}\right)^{2} .
$$

Therefore the $h$-component of the deformation $\mu \Phi+(1-\mu) \Psi$ is nondegenerate on the part $\bar{\Omega}_{1} \times \partial \Omega_{2}$ of the boundary $\partial \Omega$ whenever $R$ is sufficiently large.

Consider the $\zeta$-component of $\mu \Phi+(1-\mu) \Psi$. Since $y_{*} \neq 0$, there are positive $\tilde{\kappa}$ and $\tilde{\varepsilon}$ such that

$\rho^{2}=\left(u^{2}+v^{2}+y^{2}\right)\left(\lambda-\lambda_{0}\right)^{2} \geq \tilde{\kappa}\left(\lambda-\lambda_{0}\right)^{2}>0 \quad$ for all $\quad \zeta=(u, v, z, y) \in \bar{\Omega}_{1}(\tilde{\varepsilon})$.

Therefore $\|h\|_{C} \leq \rho^{2} R / \tilde{\kappa}$ for every point of $\bar{\Omega}$, i.e., $\|h\|_{C}=O\left(\rho^{2}\right)$ as $\lambda \rightarrow \lambda_{0}$. This relation implies $\varphi_{7}, \varphi_{8} \rightarrow 0$, which means that the $\zeta$-component of the field $\Phi-\Psi$ vanishes uniformly on $\bar{\Omega}$ as $\lambda \rightarrow \lambda_{0}$. At the same time, the point $\zeta_{*}=\left(u_{*}, v_{*}, z_{*}, y_{*}\right)$ is, by its definition, an isolated zero of the $\zeta$-component $\Theta$ of the field $\Psi$, hence for any small $\varepsilon>0$ we have

$$
\Theta\left(\zeta_{*}\right)=0, \quad|\Theta(\zeta)| \geq \kappa(\varepsilon)>0, \quad \zeta \in \partial \Omega_{1}(\varepsilon) .
$$

If such $\varepsilon$ is fixed, the $\zeta$-component of the field $\mu(\Phi-\Psi)+\Psi$ is nonzero on the part $\partial \Omega_{1} \times \bar{\Omega}_{2}$ of the boundary $\partial \Omega$ for any $\lambda$ sufficiently close to $\lambda_{0}, \lambda \neq \lambda_{0}$.

Thus, for any sufficiently large $R>0$ and any sufficiently small $\varepsilon>0$ there is a $\delta=\delta(R, \varepsilon)>0$ such that the deformation $\mu \Phi+(1-\mu) \Psi$ is a homotopy on the boundary $\partial \Omega$ of the domain $\Omega=\Omega(R, \varepsilon, \lambda)$ whenever $0<\left|\lambda-\lambda_{0}\right|<\delta$. Therefore the rotations $\gamma(\Phi, \partial \Omega)$ and $\gamma(\Psi, \partial \Omega)$ of the vector fields $\Phi$ and $\Psi$ on $\partial \Omega$ coincide. By the rotation product formula [11], the rotation of the direct sum $\Psi$ of the fields $\Theta$ and $I$ on the boundary of $\Omega=\Omega_{1} \times \Omega_{2}$ equals

$$
\gamma(\Psi, \partial \Omega)=\gamma\left(\Theta, \partial \Omega_{1}\right) \gamma\left(I, \partial \Omega_{2}\right)
$$

The rotation $\gamma\left(I, \partial \Omega_{2}\right)$ is 1 . The field $\Theta$ is differentiable at its zero point $\zeta_{*}$, i.e., $\Theta(\zeta)=\mathcal{B}\left(\zeta-\zeta_{*}\right)+o\left(\left|\zeta-\zeta_{*}\right|\right)$ where the determinant of the $4 \times 4$ matrix $\mathcal{B}$ is equal to the value $J\left(z_{*}, y_{*}\right)$ of Jacobian (28) at the point $\left(z_{*}, y_{*}\right)$. From the general theory, it follows that $\gamma\left(\Theta, \partial \Omega_{1}\right)=\operatorname{sign} J\left(z_{*}, y_{*}\right)$ whenever $\varepsilon$ is sufficiently small, therefore $\gamma(\Phi, \partial \Omega)=\gamma(\Psi, \partial \Omega)=\operatorname{sign} J\left(z_{*}, y_{*}\right)$. The inequality $\gamma(\Phi, \partial \Omega) \neq 0$ implies that $\Phi$ has at least one zero inside the domain $\Omega$.

It remains to note that for each pair of regular solutions $\left(z_{*}, \pm y_{*}\right)$ of equation (25), exactly one of them satisfies $y_{*}\left(\lambda-\lambda_{0}\right)>0$ for a given $\lambda \neq \lambda_{0}$. Therefore statements $1-4$ of Theorem 1 follow from the corresponding statements of Lemma 1. This completes the proof. 


\section{Remarks}

\subsection{Asymptotics of solutions}

The coefficient $y_{*}$ in asymptotic formula (8) is the second component of the regular solution $\left(z_{*}, y_{*}\right)$ of equation (25). The other coefficients in (8), (9) are defined by relations $q_{*} e^{i s_{*}}=(c+d i)\left(z_{*}-\tau-\alpha_{1} i\right), c_{*}=z_{*}-\beta$.

\subsection{Equations with cubic nonlinearity}

Consider equation (1) with the nonlinearity $f(x, \lambda)=x^{3}+o\left(x^{3}\right)$. Generally speaking, this equation does not have small cycles of the least period $2 \pi / w$ close to $2 \pi / w_{0}$.

To see this, let us substitute formula (14) in equation (12). Instead of (19), we obtain the system (the higher order terms are omitted)

$4 L(i w ; \lambda)=3 M(i w ; \lambda)\left(r^{2}+2\left(\xi^{2}+\eta^{2}\right)\right), 4 L(2 i w ; \lambda)=3 M(2 i w ; \lambda)\left(2 r^{2}+\xi^{2}+\eta^{2}\right)$.

Here for any $\lambda$ we have 4 real equations and only 3 unknowns: $w, r$, and $\xi^{2}+\eta^{2}$. Generally, such a system has no nontrivial solutions. More precisely,

$$
\Im \mathrm{m} \frac{L(i w ; \lambda)}{M(i w ; \lambda)}=\Im \mathrm{m} \frac{L(2 i w ; \lambda)}{M(2 i w ; \lambda)}=0
$$

implies $w=w_{0}, \lambda=\lambda_{0}$ and $r=\xi=\eta=0$.

Of course, the small cycles of the least period $\pi / w$ close to $\pi / w_{0}$ do not vanish.

\subsection{A degenerate case}

Theorems 1, 2 are not applicable if $\alpha_{1}=\alpha_{2}=0$. For example, this is the case if the linear part in (10) is independent of the parameter, i.e., equation (10) has the form

$$
L\left(\frac{d}{d t}\right) x=M\left(\frac{d}{d t}\right) f\left(x(t), x(t-\theta), x^{\prime}(t), \lambda\right) .
$$

For this equation, our main hypothesis (2) becomes

$$
L(p)=\left(p^{2}+w_{0}^{2}\right)\left(p^{2}+4 w_{0}^{2}\right) L_{1}(p)
$$


where $L_{1}\left(i w_{0} k\right) \neq 0$ for all integer $k$. Equation (41) can be studied by the method of Section 3.

Suppose the nonlinearity $f(x, y, z, \lambda)$ has the same form as in Theorem 2 . Set

$$
\varphi(\lambda)+i \psi(\lambda)=-\frac{144 w_{0}^{6}}{A(\lambda) B(\lambda)} \frac{L_{1}\left(i w_{0}\right) L_{1}\left(2 i w_{0}\right)}{M\left(i w_{0}\right) M\left(2 i w_{0}\right)}
$$

where the functions $A(\lambda), B(\lambda)$ are defined in Theorem 2 .

Theorem 3 Let $\varphi\left(\lambda_{0}\right)<0, \psi\left(\lambda_{0}\right)=0$, and let the function $\psi(\lambda)$ take values of both signs in any vicinity of the point $\lambda_{0}$. Then $\lambda_{0}$ is a HBP with the frequency $w_{0}$ for equation (41).

Under the conditions of this theorem, $\lambda_{0}$ is generally not a HBP with the frequency $2 w_{0}$. 


\section{References}

[1] H.K. Khalil, Nonlinear systems, Macmillan Publishing Company, 1992.

[2] A.I. Mees, Dynamics of feedback systems, John Wiley \& Sons, Chichester, NewYork, 1981.

[3] M. Vidyasagar, Nonlinear system analysis, Englewood Cliffs, Prentice Hall, 1993.

[4] J. Marsden, M. McCracken, Hopf Bifurcation and its Applications, Springer, New York, 1982.

[5] J. Guckenheimer, Ph. Holmes, Nonlinear oscillations, dynamical systems, and bifurcations of vector fields, Appl. Math. Sciences, 42, Springer, New York, 1990.

[6] V.I. Arnold, Geometric methods in the theory of ordinary differential equations, Springer, New York, 1983.

[7] V.S. Kozyakin, M.A. Krasnosel'skii, The method of parameter functionalization in the Hopf bifurcation problem, Nonlinear Anal. 11, 2 (1987) 149-161.

[8] A.M. Krasnosel'skii, R. Mennicken, D.I. Rachinskii, Small periodic solutions generated by sublinear terms, Report 99-009 of Institute for Nonlinear Science, National University of Ireland, University College, Cork, 1999, 1-30.

[9] K.R. Schneider, Perturbed center submanifolds and application to Hopf bifurcation. Proceedings of the $9^{\text {th }}$ International Conference on Nonlinear Oscillations (ICNO-9, Kiev-1981), 3, 450-455, Naukova Dumka, Kiev, 1984.

[10] E. Knobloch, M.R.E. Proctor, The double Hopf bifurcation with 2:1 resonance, Proc. R. Soc. Lond. A 415 (1988) 61-90.

[11] M.A. Krasnosel'skii, P.P. Zabreiko, Geometrical methods of nonlinear analysis, Springer, Berlin, Heidelberg, New York, Tokyo, 1984. 